\title{
5-fluorouracil poisoning
}

INSERM

\section{Source}

INSERM. (1999). Orphanet: an online rare disease and orphan drug data base. $\underline{5-}$ fluorouracil poisoning. ORPHA:217064

5-fluorouracil (5-FU) poisoning is a rare intoxication caused by the prolonged, low-dose administration of 5-FU, which is the mainstay of both adjuvant and advanced-disease chemotherapy regimens in colon cancer. 5-FU poisoning is characterized by gastrointestinal (nausea, emesis, diarrhea, anorexia, stomatitis) and hematologic (myelosuppression) toxicities as well as mucositis, alopecia and, occasionally, palmarplantar dysesthesia (more commonly known as hand-foot syndrome). Women have been reported to experience more 5-FU-related toxicity than men. 